\title{
Poor Doors in Erlangen
}

\author{
Umweltbezogene Mikrosegregation unter Bedingungen der Reurbanisierung
}

\author{
Klaus Geiselhart, Carolin Eisemann, \\ Fabian Feick, Stefan Kammerbauer
}

\begin{abstract}
In der sich im Zuge der Reurbanisierung verschärfenden Konkurrenz um städtische Ressourcen gestalten sich Prozesse der Segregation zunehmend kleinräumiger. Eine Fallstudie in Erlangen zeigt, wie bei den Planungen eines Quartiers im Röthelheimpark und der Rathenausiedlung bevorzugte Wohnlagen geschaffen werden, nicht zuletzt durch die Einrichtung benachteiligter Wohnlagen. Es entstehen Phänomene einer Mikrosegregation auf Quartiersebene, die über die gängigen Methoden statistischer Messung kaum noch empirisch zu erfassen sind. Die besondere Brisanz erhält das Thema durch die Tatsache, dass hierbei gesundheitliche Risiken ungleich verteilt werden, denn Umweltbelastungen werden gezielt den ärmeren Bewohner_innengruppen zugemutet, während Umweltressourcen den Wohlhabenden zugutekommen.
\end{abstract}

Ersteinreichung: 31. März 2019; Veröffentlichung online: 24. April 2020

An English abstract can be found at the end of the document.

\section{Einleitung: „Die atmen die Abgase für uns weg.“}

Derzeit lässt sich eine zunehmende sozialräumliche Polarisierung der Städte feststellen (Helbrecht 2009; Brake 2011; Häußermann 2012). Dabei werden insbesondere unqualifizierte Arbeiter_innen und bildungsferne Schichten sukzessive abgehängt (Kronauer 2002). Die Profiteur_innen dieses Wandels verlassen die unterprivilegierten Quartiere, wodurch es zu einer stärkeren Sortierung der Wohnbevölkerung nach Einkommen kommt - die Segregation der Städte nimmt zu (u. a. Helbig/Jähnen 2018). Die zunehmende soziale Ungleichheit überlagert sich zudem mit einem Rückzug des Staates aus der Wohnungsversorgung (Häußermann 2012). Die Objektförderung wurde zu Gunsten der Subjektförderung, also der sozialstaatlichen Unterstützung bedürftiger Personen mit Wohngeld, reduziert. Während die einen dies kritisch als indirekte Subventionierung von Wohnungseigentümer_innen betrachten (Häußermann 2012), beschwören die anderen Schreckensszenarien langsamer Verelendung benachteiligter Quartiere, welcher nur mittels einer Aufwertung durch private Investor_innen begegnet werden könne (Lojewski 2013). Demnach haben Immobilienbesitzer_innen ein besonderes Interesse an der Aufwertung von Quartieren, denn eine soziale Profilierung des Wohngebietes bewirkt auch eine Aufwertung ihrer Immobilien auf dem Wohnungsmarkt. In dieser Auffassung ist Gentrifizierung ein probater Motor 
der Stadt- und Quartiersentwicklung, mit dem positiven Nebeneffekt, dass durch Zuzug finanzstarker Bewohner_innen in vormals marginalisierte Stadtgebiete gleichzeitig auch soziale Mischung gefördert und Segregation reduziert wird. Zudem finden, dieser Auffassung entsprechend, Gentrifizierungsprozesse meist nur relativ punktuell statt, weswegen sich auch kaum Verdrängungseffekte für benachteiligte Bevölkerungsgruppen ergeben. Dieser Einschätzung widersprechen Befunde von Verdrängung und unfreiwilliger Segregation, also des Umzuges von Geringverdiener_innen wegen mangelnder Wahlmöglichkeit des Wohnstandortes, verursacht durch die Bedingungen des Wohnungsmarktes (u. a. Häußermann 2012; Helbrecht 2016).

Prozesse der Reurbanisierung, wie Gentrifizierung, Nachverdichtung und Aufwertung innenstadtnaher Gebiete machen den städtischen Raum zusehends knapper und bewirken eine verschärfte Konkurrenz um städtische Ressourcen (Brake 2011). Segregation stellt sich im Zuge der sozioökonomischen Polarisierung uneindeutig, zunehmend dynamischer und kleinräumiger dar. Quartiersbewohner_innenschaften erleben widerstreitende Tendenzen der Durchmischung und Verdrängung. Stadtverwaltungen fördern bewusst soziale Mischung, um negative Folgen von Segregation (,Quartierseffekte') zu verringern und der Entstehung ,sozialer Brennpunkte، Einhalt zu gebieten (Faßmann/Franz 2005; Harlander 2012), wie das derzeit in Deutschland, Frankreich und Polen für einige Großwohnsiedlungen beschrieben werden kann (Brailich et al. 2008). Es gilt, eine ,Ghettoisierung، bestimmter Bevölkerungsgruppen zu verhindern, wie sie insbesondere für afroamerikanische Bevölkerungsgruppen in den USA beschrieben wurde (z. B. Massey/Denton 1993). Es zeigt sich aber, dass soziale Mischung nicht auch automatisch zu einer sozialen Durchmischung im Sinne eines interaktiven Zusammenlebens der verschiedenen Bevölkerungsgruppen untereinander führt (Faßmann/Franz 2015; Lees 2008; Bacqué/Fijalkow 2012). Räumliche Nähe allein bewirkt keine lebendigen Nachbarschaften.

Indes kann soziale Trennung durch Architektur gezielt auf ganz kleinem Raum hergestellt werden, beispielsweise mittels des in Manhattan, Brooklyn oder London entstandenen Phänomens der poor doors (Meubrink 2016). Gemeint sind separate Eingänge zu Wohnungen mit erschwinglichen Mieten in den unteren Etagen von Luxustowern: Diejenigen, die es sich leisten können, treten in einen hochwertigen Eingangsbereich mit Lobby und Portier ein, um von dort zu den hochpreisigen Wohnungen in den oberen Etagen zu gelangen - auf den niedriger gelegenen Wohnebenen hingegen leben die Armen, die eine schmucklose ,Hintertüre' auf der Rückseite des Gebäudes benutzen müssen, um zu ihren Wohnungen zu gelangen. Die Verkehrswege und Aufenthaltsbereiche der Bewohner_innengruppen sind streng voneinander getrennt. Eine Durchmischung ist nicht gewollt. Die unteren Etagen sind weniger attraktiv und werden schlechter ausgestattet. Die Bauträger_innen erhalten durch die Errichtung von Sozialwohnungen Steuervergünstigungen, bessere Zinsen und die Erlaubnis, höher und größer zu bauen.

Derartige Phänomene kleinräumiger Segregation werden in tagesaktuellen Medien teils scharf kritisiert (Pitzke 2014; Licea 2016) und haben damit im angloamerikanischen Sprachraum öffentliche Diskussionen entfacht (z. B. NYU Furman Center 2015). Die Debatten entzünden sich an der Frage, ob die Zahl der Eingänge wichtiger sei als die Qualität der entstehenden 
Wohnungen, schließlich seien wesentlich schlechtere Quartiere bekannt. Sei es für ärmere Bevölkerungsgruppen nicht auch ein Vorteil, in eine gehobene Nachbarschaft ziehen zu können und würden Städte durch gemischte Nachbarschaften letztlich nicht auch gerechter werden? Diese Debatte erinnert sehr an die oben skizzierte deutschsprachige Debatte um soziale Mischung und Gentrifizierung. Poor doors allerdings scheinen in Deutschland unvorstellbar oder werden zumindest nicht diskutiert.

Im Folgenden wollen wir anhand zweier innenstadtnaher Nachbarschaften in Erlangen zeigen, dass auch hier ähnliche Segregationsprozesse stattfinden. Das qualitative Vorgehen dieser Fallstudie erlaubt es, ein zentrales Merkmal zu identifizieren, anhand dessen bevorzugte von benachteiligten Wohnlagen unterschieden werden, welches in der englischsprachigen Debatte zu poor doors bisher aber keine Rolle zu spielen scheint. Wir werden zeigen, wie sich in beiden Fällen, bei der Nachverdichtung in der Rathenau ebenso wie bei der Planung eines neuen Quartiers im Röthelheimpark, die festgestellte Mikrosegregation auf Quartiersebene als eine Frage der Umweltgerechtigkeit darstellt. Umweltbedingte Belastungen und Umweltressourcen werden nicht gleichmäßig auf alle Bewohner_innen verteilt. Für die Qualität eines Wohnstandortes spielt vor allem die Lage bezüglich viel genutzter Verkehrsachsen sowie zu Grünflächen eine entscheidende Rolle. Wir werden zeigen, wie die Belastungen und Ressourcen dabei durch gezielte Planung der Wohneinheiten graduell entsprechend der finanziellen Leistungsfähigkeit der Bewohner_innen verteilt werden. Vielfach werden Sozialwohnungen als Riegel entlang besonders belasteter Achsen errichtet und dienen quasi als Schutzschild. „Die atmen die Abgase für uns weg“, wurde hierzu von einem unserer Interviewpartner_innen kommentiert. Eine besondere Brisanz erhält die Thematik dadurch, dass mit dieser Art der Segregation erhebliche Gesundheitsgefährdungen einhergehen. Ferner wird deutlich werden, dass die beschriebenen Prozesse so kleinräumig sind, dass sie mit gängigen statistischen Verfahren kaum darstellbar sind, wodurch die schwerwiegenden Folgen für die Betroffenen für systematische Erhebungen quasi unsichtbar sind. Aufgrund dieser statistischen Unsichtbarkeit der hier beschriebenen Segregation und der Tatsache, dass sie sich an Umweltbedingungen ausrichtet, sprechen wir von einer umweltbezogenen Mikrosegregation.

\section{Methoden}

Als qualitative Fallstudie erhebt diese Untersuchung nicht den Anspruch, die hier aufgezeigten Prozesse als allgemeingültige Prinzipien zu behaupten. Vielmehr geht es darum, am konkreten Beispiel aufzuzeigen, anhand welcher Prozesse sich Strukturen der Ungleichheit ausbilden. Untersucht wurden die einstmals von Siemens für Mitarbeiter_innen errichtete Rathenausiedlung, die in Teilen aktuell nachverdichtet wird, und eine vollkommen neu geplante und gebaute Nachbarschaft im Stadtteil Röthelheimpark. Die Quartiere wurden mit einem jeweils leicht angepassten Methodenset untersucht. $\mathrm{Zu}$ Beginn wurden Sozialraumbegehungen durchgeführt - im Röthelheimpark ohne Schlüsselpersonen, in der Rathenausiedlung mit den ehemaligen Leitern der GBW-Mietergemeinschaft. Ziel war die Erhebung räumlicher und sozialer Strukturen der Untersuchungsgebiete, der jeweiligen Wohnbebauung und 
des Charakters der zur Verfügung stehenden Wohneinheiten. Ausgewertet wurden die Sozialraumbegehungen anhand fotographischer Aufnahmen, Skizzen und Notizen. Einen wesentlichen Teil der Untersuchung bildeten zwölf qualitative Interviews mit unterschiedlichen Akteuren. Es kamen Expert_inneninterviews und Interviews mit Bewohner_innen zum Einsatz, die in Anlehnung an die qualitative Inhaltsanalyse nach Mayring (2015) ausgewertet wurden. Zu den Expert_innen zählten Fachkundige aus der Erlanger Stadtplanung, Politiker_innen sowie Mitarbeiter_innen sozialer Verbände (Bürgertreff, Kirche). Dadurch konnten die Aussagen der Expert_innen den Aussagen der betroffenen Bewohner_innen gegenübergestellt werden. Ergänzend wurden Informations- und Bürgerbeteiligungsveranstaltungen besucht und die Debatten um die jeweiligen städtebaulichen Entwicklungsmaßnahmen wurden mittels Internet- und Zeitungsrecherchen nachgezeichnet. In der Rathenausiedlung bestand zudem die Möglichkeit, an Bürger_innensprechstunden teilzunehmen sowie an Veranstaltungen, die von der örtlichen Mieter_innengemeinschaft organisiert wurden. Besuche der Auftaktveranstaltung des städtischen Projektes „Bezahlbarer Wohnraum - Lebenswerte Stadt“ am 16.03.2018 sowie der Fachtagung „Gesundheit und Stadtplanung" am 25.07.2018 lieferten gehaltvolle Hinweise zur Kontextualisierung der Teilstudien.

\section{Umweltgerechtigkeit, Gesundheit und Segregation}

Ebenso wie der Begriff, poor doors'stammt auch ,Umweltgerechtigkeit' (environmental justice) aus dem Amerikanischen. Geprägt wurde der Begriff während der politischen Auseinandersetzungen um den Bau einer Mülldeponie in einem kleinen Ort in North Carolina zu Beginn der 1980er Jahre. Dort lebten rund ein Drittel African Americans, die dem Vorhaben Widerstand entgegenbrachten. Zwar wurde die Mülldeponie trotzdem gebaut, doch griff die Bürgerrechtsbewegung die Thematik auf nationaler Ebene auf und verknüpfte sie mit Fragen des Umweltschutzes. Neu war die Erkenntnis, dass Umweltrisiken auch innerhalb hochindustrialisierter und demokratisch verfasster Länder ungleich verteilt sein können. Heute stellt sich Umweltgerechtigkeit für die Geographie als ein sozial relevanter Ansatz zur Analyse lokalisierter Mensch-Umweltbeziehungen dar (Flitner 2003).

Allgemein gesprochen beschreibt Umweltgerechtigkeit Fragen zur sozialen und räumlichen Verteilung von Umweltbelastungen und -ressourcen. Gabriele Bolte et al. (2012: 23) zufolge reicht der Begriff „von einem Verständnis von Umwelt als Risiko über die Nutzungschancen von Umwelt als Ressource bis hin zur gerechten beziehungsweise ungerechten Verteilung der Vorund Nachteile, die aus der Umweltnutzung hervorgehen“. In Städten stellt sich die Frage der Umweltgerechtigkeit vor allem in Bezug auf Belastungen von Böden, Wasser und Luft. Von Bedeutung für einen Wohnstandort ist demnach die Qualität der ihn umgebenden Umweltbedingungen. Die Hauptfaktoren hierbei sind im positiven Sinne die Nähe und der Zugang zu urbanen Erholungsräumen wie Grünräumen oder Gewässern und im negativen Sinne eine Nähe zu den meist linienförmigen Belastungen entlang der Verkehrsachsen durch Lärm und Abgase (Bolte et al. 2012). Sogenannten environmental goods stehen environmental bads gegenüber. 
Eine besondere Brisanz erhalten Fragen der Umweltgerechtigkeit unseres Erachtens aber erst durch eine Berücksichtigung von Gesundheit, also in Anbetracht der Tatsache, dass Umweltbedingungen sowohl gesundheitsverletzende wie auch gesundheitsfördernde Wirkungen entfalten können. Allgemeiner wird unter Umweltgerechtigkeit demnach die Zusammenführung der Faktoren soziale Lage, Umwelt und Gesundheit verstanden (vgl. Böhme/ Bunge/Preuß 2016: 141). Es geht also nicht nur um eine Ungleichverteilung von Besitz und Komfort, sondern um die Zumutung verminderter Lebensqualität, gesundheitlicher Risiken und umweltbedingter Krankheiten. In diesem Sinne sollen hier relevante umweltbedingte gesundheitliche Auswirkungen kurz dargestellt werden.

\subsection{Umweltbedingte Einflüsse auf Gesundheit und Krankheit}

Grünanlagen und Gewässer, sogenannte environmental goods, stellen eine wichtige Voraussetzung für die Lebensqualität, Gesundheit und das Wohlbefinden der Bevölkerung dar und besitzen gesundheitsförderliche Potenziale (Claßen/Heiler/Brei 2012; Kowarik/Bartz/Brenck 2016). Stadtgrün und Stadtblau fördern die physische Gesundheit indem sie Ökosystemleistungen übernehmen: Naturnahe Bereiche, Grünanlagen, aber auch einfache Grünstreifen verbessern das Stadtklima, fördern den Klimaschutz, sorgen für saubere Luft, mindern Lärm und sichern die Funktionsfähigkeit von Böden und Gewässern. Zudem fördern sie die physische Gesundheit dadurch, dass sie als Bewegungsräume fungieren (Kistemann/Völker/Lengen 2010; Kowarik/ Bartz/Brenck 2016). Darüber hinaus hat die grün-blaue Infrastruktur allein durch ihre Präsenz schon positive psycho-physische Einflüsse: Sie wirkt stressreduzierend, blutdrucksenkend, konzentrationssteigernd und erholsam. Insgesamt steigert Stadtgrün das Wohlbefinden und wirkt damit präventiv bezüglich psychischer Erkrankungen (Claßen/Heiler/Brei 2012; Kowarik/Bartz/Brenck 2016). Nicht zuletzt sind Stadtgrün und Stadtblau von sozialer Bedeutung: Als öffentliche, frei zugängliche Begegnungsräume ermöglichen sie eine soziale Durchmischung unterschiedlicher Gruppen und fördern damit Wohlbefinden und soziale Integration.

Wie schon angedeutet, stehen diesen environmental goods auch environmental bads gegenüber: Der Mensch belastet die Umwelt erheblich, sodass Boden, Wasser und Atmosphäre enorme Schäden davontragen. Zu den Bodenbelastungen anthropogenen Ursprungs zählen beispielsweise die Verunreinigung durch Schadstoffe und die Flächenversiegelung. Altlasten beziehungsweise altlastenverdächtige Flächen spielen dabei eine wichtige Rolle. Darüber hinaus wird Wasser durch Abwässer oder Verschmutzung (Industrie, Landwirtschaft, Erdöl, Plastikmüll etc.) beeinträchtigt - auch die Luftqualität kann durch diese Einflussfaktoren mit Schadstoffen belastet werden.

Gesundheitlich relevant sind aber auch Lärmemissionen. Dabei ist der Straßenverkehrslärm in deutschen Städten als Hauptlärmquelle einzustufen. Neben den Langzeitfolgen wie Gehörschäden, Herz-Kreislauf-Erkrankungen, Arteriosklerose oder Bluthochdruck wirkt sich Lärmbelastung auch negativ in Form von Stress und Schlafstörungen aus (Umweltbundesamt 2015, 2017).

Als weitere gesundheitliche Belastungen gelten Verunreinigungen der Luft: Wesentlich sind Stickstoffdioxid NO2 und Feinstaub PM10 sowie PM2,5. Viele 
Städte sind aufgrund hoher Emissionen einer starken Luftschadstoffbelastung ausgesetzt, wobei die höchsten NO2- und Feinstaubkonzentrationen ganz klar an viel befahrenen Straßen festzustellen sind (Umweltbundesamt 2018b). Gesundheitsgefahren treten durch das ätzende Reizgas NO2 vor allem in Form von geschädigtem Schleimhautgewebe und Reizungen der Augen auf. Besonders betroffen sind Menschen mit vorgeschädigten Atemwegen und Allergien. Zudem führt eine erhöhte NO2-Konzentration zu Herz-Kreislauf-Erkrankungen. Bei Feinstaub ist die gesundheitsschädigende Wirkung von der Größe und Eindringtiefe der Partikel abhängig. Es kommt zu Schleimhautreizungen, lokalen Entzündungen im Rachen, der Luftröhre und den Bronchien. Ebenso verursacht Feinstaub eine erhöhte Thromboseneigung oder wirkt sich auf die Herzfrequenzvariabilität aus (Umweltbundesamt 2018a).

\subsection{Die Messbarkeit umweltbezogener Segregation}

Dahlgren und Whitehead (1992) haben die hier genannten und weitere lebensweltliche Determinanten von Gesundheit für die WHO systematisiert. Ihr Modell wurde von Barton und Grant (2006: 253) zu einer health map für Lebensräume weiterentwickelt, die es ermöglicht, psychische, soziale und kulturelle Determinanten von Gesundheit auf kleinräumiger Ebene zu betrachten. Beide Modelle finden heute in der regionalen Planung von Gesundheit Anwendung (Claßen/Mekel 2016; LZG.NRW 2016). Bei kleinräumiger Betrachtung wird deutlich, dass die genannten Umweltbelastungen und Umweltressourcen in städtischen Gebieten häufig räumlich und sozial ungleich verteilt sind und regional unterschiedliche Qualitäten aufweisen können (Claßen/Heiler/Brei 2012) und es stellt sich die Frage, inwiefern eine soziale umweltbezogene Segregation stattindet.

Klassischerweise wird unter ,Segregation“ die Konzentration von unterschiedlichen Bevölkerungsgruppen in unterschiedlichen Teilräumen der Stadt verstanden. Soziale Segregation bezeichnet entsprechend eine räumliche Trennung von Arm und Reich (Farwick 2012; Helbig/Jähnen 2018). Dabei präsentierte sich Segregation zu Zeiten der Chicagoer Schule noch als Prozess der Konzentration benachteiligter Bevölkerungsgruppen in weitgehend homogenen Zonen oder Sektoren. Entsprechend wurden Analysen auf Stadtteil-, census tract- oder Postleitzahlenebene lange Zeit als ein probates Mittel angesehen, derartige Konzentrationsprozesse abzubilden. Auch heute wird mittels klassischer Sozialraumanalysen oder indikatorenorientierter Sozialberichterstattung (Aehnelt 2011) versucht, Sozialraumstrukturen in diesen Maßstäben zu evaluieren. Zwar wird auch auf diesen Ebenen eine Zunahme der Segregation festgestellt (Helbig/Jähnen 2018), doch gibt es auch Hinweise auf Differenzierungen innerhalb solcher Einheiten. So stellen beispielsweise Kabisch et al. (2018) im Falle von Leipzig-Grünau, einer Großwohnsiedlung, die sich über fünf Ortsteile erstreckt, fest, dass sich diese Ortsteile trotz homogener Gebäudekubatur in demographischer, sozialstruktureller Hinsicht sowie bezüglich des Images unterscheiden. Darüber hinaus zeigen sich noch kleinräumigere Differenzierungen innerhalb dieser Ortsteile. All diese Differenzierungen lassen sich, so die Autor_innen, auf langfristige Dynamiken des Wohnungsmarktes, kleinräumige Ausschöpfungen von 
Entwicklungspotenzial bestimmter Lagevorteile, damit verbundene Imagekampagnen und daraus entsprechend resultierende kleinräumige Milieubildungsprozesse zurückführen. Dabei seien insbesondere die kleinsträumigen Differenzierungen besonders hilfreich, „um Veränderungen und Herausforderungen identifizieren zu können" (Kabisch et al. 2018: 140).

Jekleinräumiger Daten erfasst und aggregiert werden, desto besser können auch Ungleichheiten abgebildet und Nachbarschaftseffekte identifiziert werden (Goebel/Hoppe 2015: 26 ff.). Diesem Anspruch stehen aber oftmals Probleme bezüglich der Datenverfügbarkeit im Wege. Methodisch kann dieser Problematik Rechnung getragen werden, indem dort, wo keine kleinräumigen Daten vorliegen, mittels eines Homogenitätsindex das Maß der den Untersuchungseinheiten inhärenten Ungleichheiten dargestellt wird (Zehner 2004). Akkurater aber ist es, wenn die statistischen Ämter der Städte ihre statistischen Einheiten den lebensweltlichen Gegebenheiten anpassen und damit aussagekräftigere Daten zur Verfügung stellen. So wurde im Land Berlin, um ein substanzielles Umweltgerechtigkeitsmonitoring zu ermöglichen, die amtliche Statistik auf 447 Planungsräume, sogenannte lebensweltlich orientierte Räume (LOR), umgestellt (Bömermann/Jahn/ Nelius 2006; Klimeczek 2014). Dabei wurden unter anderem Gebiete gleicher Gebäudekubatur von denen mit anderen Bebauungsarten getrennt, wie etwa Großwohnsiedlungen von Einfamilienhaussiedlungen, die bisher vielfach in einer statistischen Einheit verbunden waren. Hierdurch werden die unterschiedlichen Realitäten in den Quartieren besser statistisch abgebildet.

Nun muss aber gesehen werden, dass Leipzig-Grünau für 85.00o Einwohner geplant war und heute dort etwa 42.000 leben. Auch die Berliner LORs sind mit rund 7.500 Einwohner_innen (Bömermann et al. 2006: 369) deutlich größer als unsere Untersuchungsgebiete. Wir betrachten im Folgenden Nachbarschaften innerhalb von Stadtteilen, die als Ganze nur 6.368 Wohnberechtigte im Röthelheimpark und 7.337 im Anger (hier liegt die Rathenausiedlung) aufweisen (Stadt Erlangen 2019). Die betrachteten Nachbarschaften sind noch einmal deutlich kleiner. Teilweise beziehen wir uns in der Untersuchung auch auf einzelne Gebäudezeilen. Ist die Datenverfügbarkeit auf kleinräumiger Ebene schon schwierig, weil die Städte bei der Bereitstellung sehr zurückhaltend sind (Goebel/Hoppe 2015: $23 \mathrm{ff}$.), so ist sie in unserem Falle nahezu unmöglich. Da sich manche unserer Bezugseinheiten tendenziell auf sehr wenige Individuen beschränken, wären datenschutzrechtliche Probleme vorgezeichnet.

\subsection{Soziale Mischung, Segregation und politische Steuerung}

Aber Segregation ist nicht allein ein statisches Phänomen. „In einer dynamischen Betrachtung werden unter Segregation Prozesse der räumlichen Differenzierung, Sortierung und Separierung gefasst.“ (Farwick 2012: 381) Die Herausbildung homogener ortsbezogener Milieus ist aber kein natürlicher Prozess, sondern Ausdruck sozialer Verhältnisse (Belina 2011). Um eine Konzentration benachteiligter Bevölkerungsgruppen zu vermeiden, fördern deutsche Städte deswegen soziale Mischung. Harlander (2012) beschreibt vielfältige Maßnahmen, die Städte ergreifen, um eine Konzentration benachteiligter Bevölkerungsgruppen zu vermeiden, einige davon auch, „obwohl 
sie strenggenommen diskriminierend sind“ (ebd.: 52). In den Stadtteilen soll eine vielfältige Bewohner_innenschaft unterschiedlicher ethnischer Herkunft, Religion, Geschlecht, Alter oder Behinderung leben. Das soll die Lebensqualität für alle Stadtbewohner_innenin gleichem Maßegewährleisten - „Soziale Mischung erscheint als Ideal“ (Faßmann/Franz 2005: 195).

Segregation gilt also in der Regel als ein nach Möglichkeit zu vermeidender Prozess. Als Schreckgespenst werden oftmals sogenannte Nachbarschafts- oder Quartierseffekte beschworen (Bürkner 2011; Häußermann 2012; Dangschat 2017). Demnach bilden sich lokale Milieus, die eine Abwärtsspirale in Gang setzen. Es wird davon ausgegangen, dass ein heruntergekommenes Wohnviertel mit schlechtem Image nicht nur das Verhalten seiner Bewohner_innenschaft prägt, sondern auch die Entwicklungsmöglichkeiten der Einzelnen, insbesondere der Kinder und Jugendlichen, massiv einschränkt, wodurch Armut sozusagen ,vererbt' wird. Dabei wird aber häufig vernachlässigt, dass lokale Milieus auch eine Ressource der Lebensbewältigung darstellen können (Häußermann 2012; Cheshire 2012). In diesem Sinne werden auf theoretischer Ebene Intersektionalitätsperspektiven vorgeschlagen, die sich der Heterogenität von Lebenslagen und Lebenschancen in den Stadtteilen und Nachbarschaften zuwenden (Bürkner 2011). Auch führt soziale Mischung keineswegs automatisch dazu, dass sich die verschiedenen Bewohner_innengruppen einer Nachbarschaft auch alltagsweltlich begegnen und austauschen, es also auch zu sozialer Durchmischung kommt (Faßmann/ Franz 2005; Lees 2008; Bacqué/Fijalkow 2012). So stellt ein sozial gemischtes Quartier auch keine Lösung für Armutsproblematiken, Ausgrenzung und Diskriminierung dar (Harlander/Kuhn 2012). Politische Versprechungen, wonach Gentrifizierungsprozesse soziale Mischung bewirken, seien, so Loretta Lees (2008), eher kosmetischer Natur, denn diese Prozesse verliefen naturgemäß in Richtung Segregation und Polarisierung. Auch Ilse Helbrecht (2009) erkennt ein Streben von Mittelstandsbewohner_innen gentrifizierter Nachbarschaften zur Herausbildung homogener Enklaven und sieht dadurch die Hoffnung enttäuscht, mit der Reurbanisierung in der Wissensgesellschaft könne es zu einer offeneren und integrativen Stadtgesellschaft kommen. Im Zuge der Reurbanisierung bildet sich Klaus Brake (2011) zufolge eine „tendenziell aggressive Konkurrenz“ um die Aneignung des Städtischen heraus, „mit einem eher egoistischen Verhältnis dazu, wem die Stadt gehört“ (ebd.: 88).

Eine gängige städtebauliche Maßnahme zur Förderung sozialer Mischung, sowohl in Großbritannien als auch Deutschland, ist die Auflage bei größeren Bauprojekten und der Neu- und Umgestaltung von Stadtteilen, einen bestimmten Anteil an Sozialwohnungen zu schaffen (Faßmann/ Franz 2005: 195). Nun ist es aber naheliegend, dass Bauträger_innen Ausstattung und Lage preisgünstiger Wohnungen bewusst und nach ökonomischen Gesichtspunkten gestalten. Im Falle der poor doors - diese lassen sich auch als das Ergebnis derartiger städtischer Wohnungspolitik betrachten (Meubrink 2016) - werden bewusst sehr kleinräumige Ungleichverteilungen etabliert, ohne dass soziale Durchmischung überhaupt angestrebt wird. Dabei ist bisher kaum wissenschaftlich reflektiert, an welchen Bedingungen und Kriterien sich derart kleinräumige Differenzierungen schließlich ausrichten. Die vorliegende Untersuchung spürt derartigen Prozessen in zwei Quartieren nach. 


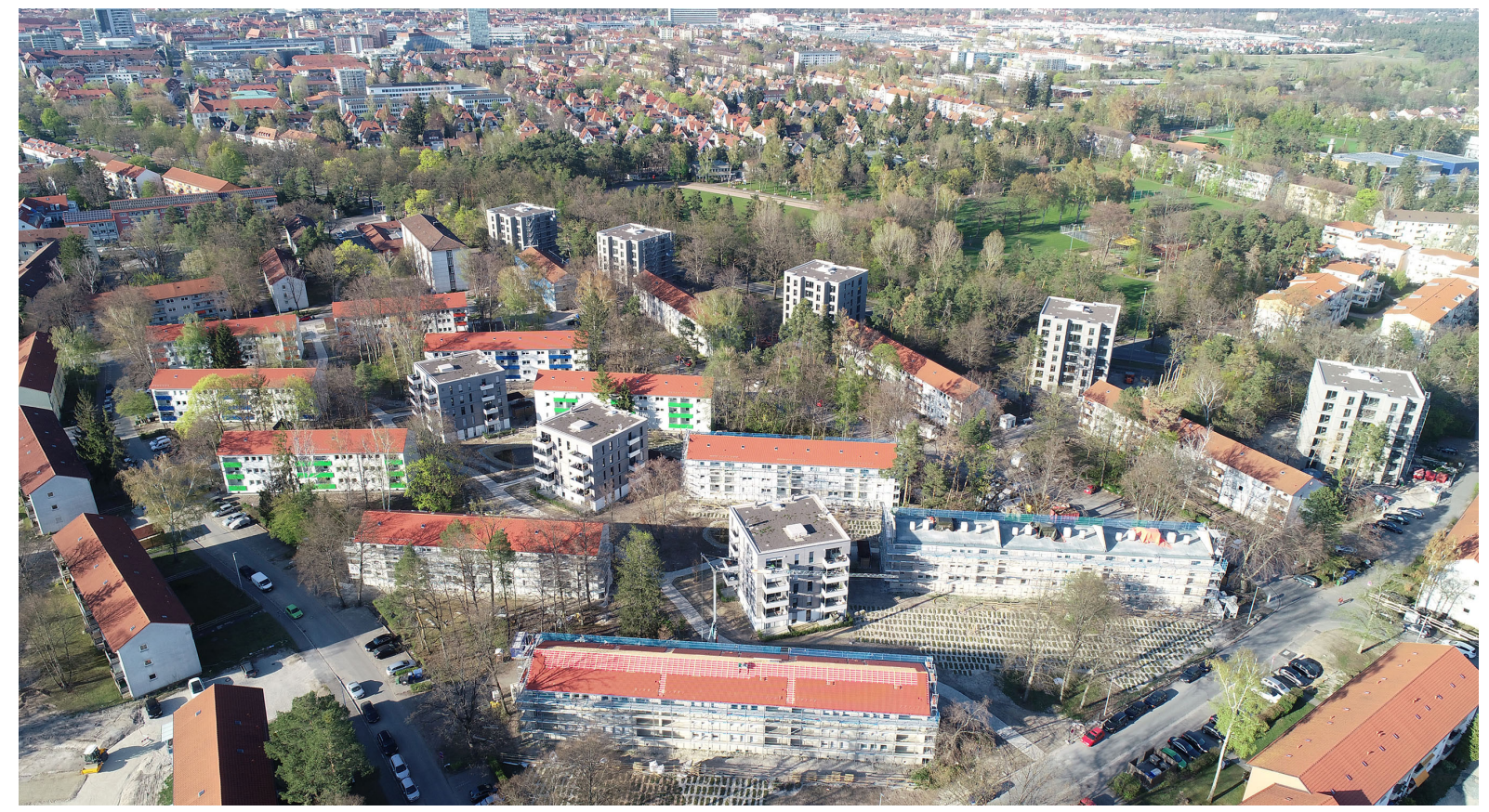

Abb. 1 Luftbild der Nachverdichtung in der Rathenausiedlung (Foto: Jan Gemeinholzer)

\section{Nachverdichtung in der Rathenau}

Die Rathenausiedlung - wie die Bewohner_innen ihr Quartier nennen - liegt im Süden Erlangens zwischen der Nürnberger Straße, der Stintzingstraße und der Paul-Gossen-Straße. In den 1960er Jahren wurde sie vom SiemensKonzern als klassische Arbeitersiedlung, mit den für die 6oer bis 7oer Jahre typischen dreigeschossigen Zeilenbauten mit Satteldach, errichtet (Abbildung 1). Die großzügige Anlage bot weite Freiflächen zwischen den Häusern, welche vor den Nachverdichtungsarbeiten durch einen losen Baumbestand aus damals recht alten Bäumen gekennzeichnet war. In einem Interview wurde auch von einer „Siedlung im Wald“ gesprochen (Interview Mitarbeiter_in Stadtplanung Erlangen, 24.05.2018). Die Freiflächen boten, neben mehreren Spielplätzen, Platz für zahlreiche weitere Aufenthaltsund Sitzmöglichkeiten zugunsten unterschiedlicher Altersklassen und Bedürfnisse (Interview Bewohner_innen Rathenau 1, 27.06.2018).

\subsection{Planungsprozess der Rathenausiedlung}

Nachdem ein Teil dieses Quartiers im Frühjahr 2009 an die GBW-Gruppe (heute Dawonia) veräußert wurde, entschied diese, den Bestand der erworbenen Liegenschaften einer Nachverdichtung zu unterziehen. 2015 initiierte sie einen Architektenwettbewerb, nachdem im Frühjahr desselben Jahres ein Anwohner_innen-Workshop und mehrere Voruntersuchungen stattgefunden hatten. Nach den Bebauungsplanverfahren (2016-2017) und den Baugenehmigungen (2017-2018) wurde 2018 mit den ersten Baumaßnahmen begonnen. Das Projekt soll 2021 abgeschlossen sein. Die GBWGruppe versprach eine energetische und altersgerechte Modernisierung der Bestandswohnungen, ohne eine Kernsanierung vorzunehmen. Das Quartier sollte im Ganzen aufgewertet werden. Laut damaligem Internetauftritt der GBW sollten in etwa 300 bis 400 neue Wohneinheiten durch Aufstockung und Neubau geschaffen werden (GBW o. J.a). Bei einer Mieter_inneninformationsveranstaltung im April 2018 war hingegen von einer 
„Nachverdichtung durch Neubauten mit mehr als 640 Wohneinheiten“ die Rede(GBW 2018: 6). Damit hatte sich die Zahl der geplanten Wohnungen gegenüber der öffentlichen Bekanntgabe nahezu verdoppelt. Die Bewohner_innen der Rathenausiedlung befürchten aber eine noch höhere Zahl an Neubauwohnungen. Grund hierfür ist unter anderem ein Zeitungsartikel aus den Erlanger Nachrichten. Dort wird im Februar 2018 der frühere Bereichsleiter der Projektentwicklung der GBW zitiert, wonach nun mit dem Bau von 950 Wohnungen begonnen werden solle (vgl. Erlanger Nachrichten 10.02.2018).

Teilweise werden die neuen Wohnungen in sieben Punkthäusern Platz finden. Bei diesem Gebäudetyp liegen die Wohnungen ringförmig um einen innenliegenden Treppenhauskern, wodurch sich in vorliegenden Fällen ein quadratischer Grundriss ergibt. Dem Bebauungsplan kann entnommen werden, dass diese Bauwerke die Größe der meisten bestehenden Häuser im Quartier überschreiten. Tatsächlich wirken sie im Verhältnis zum Altbestand eher wuchtig (Abbildung 1) und, wie die Anwohner_innen nicht müde werden zu erwähnen, „fehl am Platz“ (Interview Bewohner_innen Rathenau 1 und 2, 27.06.2018 und 29.06.2018). In der Mitte des Quartiers (Abbildung 2: Signatur C) entstehen drei fünfstöckige Punktbauten mit jeweils 13 Wohneinheiten, entlang der Nürnberger Straße fünf siebengeschossige Bauten mit je 28 Wohnungen. Weitere Wohnungen entstehen in zusätzlichen Zeilenbauten (Abbildung 2: Signatur A, B, D), von denen einige, wie im Folgenden beschrieben, dem „Schallschutz“ dienen sollen.

\subsection{Wohnblöcke als Schallschutzmaßnahme}

Fast schon zynisch wirkt auf die Bewohner_innen, dass die GBW als Maßnahmen zur Aufwertung des Quartiers „Schallschutzmaßnahmen in Teilbereichen durch zusätzliche Bebauung “vorsieht (GBW o. J.a). Damit ist der Bau der Wohnblöcke gemeint, die entlang der beiden vielbefahrenen Straßen entstehen sollen (Abbildung 2: Signatur A und B). Zwei im Bebauungsplan Nr. 345 als „Schallschutzriegel“ (Stadt Erlangen 2018) bezeichnete Bauten sollen die dahinterliegenden Wohnlagen von der Belastung durch die Nürnberger Straße und die staugefährdete Kreuzung Nürnberger Straße, Gebbertstraße und Paul-Gossen-Straße abschirmen. In diesen Wohnungen werden 75 Sozialwohnungen entstehen (GBW 2018). Im Süden werden zur besseren Abschirmung der vierspurigen Paul-Gossen-Straße sogar drei giebelständige Wohnblocks abgerissen und etwas weiter nördlich neu errichtet, damit zur Straße hin ein traufständiger Wohnblock Platz findet. Da die Stadt bei Stadtentwicklungsprojekten nach den EOFRichtlinien 25 Prozent geförderte Wohnungen vorschreibt, fehlen bei 640 Neubauwohnungen etwa 85 weitere geförderte Wohneinheiten. Die Bewohner_innen sind davon überzeugt, dass diese in diesem zweiten „Schallschutzriegel" entlang der Paul-Gossen-Straße geschaffen werden (Interview Bewohner_innen Rathenau 2, 29.06.2018). Ihrer Ansicht nach werden die geförderten Wohnungen zur Profitmaximierung als Schall- und Dreckschutz an den Hauptverkehrsstraßen errichtet. Die Punktbauten in der Mitte des Quartiers, abseits von Lärm und Dreck, stellen hingegen die privilegierten Wohnlagen dar, wodurch sich hier, so die Vermutung der Bewohner_innen, höhere Mietpreise erzielen lassen. 


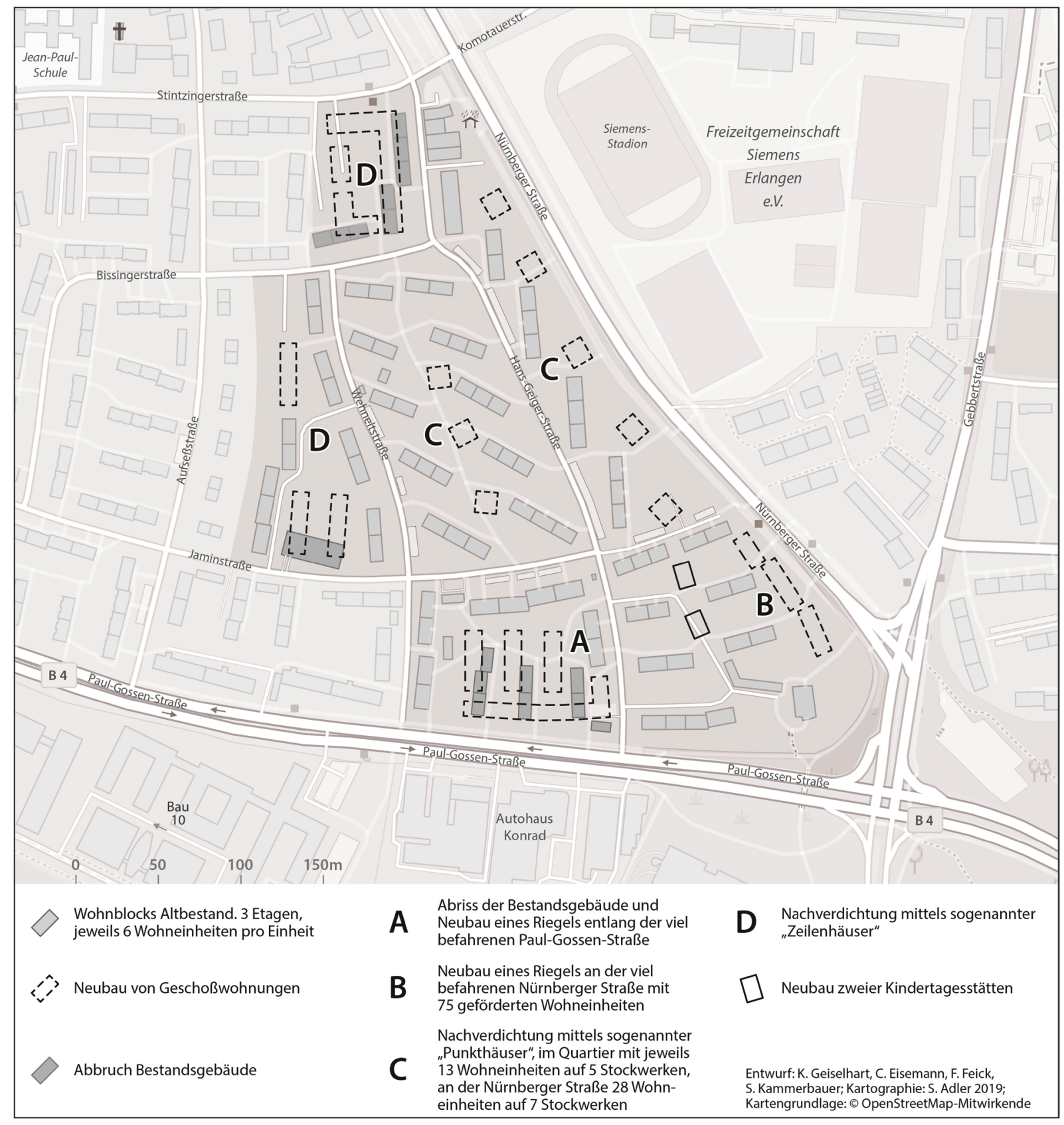

Abb. 2 Veränderungen im Wohnbaubestand im Zuge der Nachverdichtung der Rathenausiedlung (Quelle: Eigene Darstellung, Kartographie: Stephan Adler)
Die Stadtplanung dementiert diese Auffassung auch keineswegs: „Geförderter Wohnungsbau findet aus wirtschaftlichen Gründen häufig an verkehrsbelasteten Straßen statt. Dieser darf bestimmte Preise nicht übersteigen, da Grund und Boden auch seinen Preis hat." (Interview Mitarbeiter_in Stadtplanung Erlangen, 24.05.2018) Die daraus resultierende gesundheitliche Belastung der Bewohner_innen von Sozialwohnungen wird von der Planung augenscheinlich geduldet oder gar vorgesehen.

\subsection{Informationspolitik und Effekte der Nachverdichtung}

Leider kann hier aus Platzgründen nur kurz auf das taktische Vorgehen der GWB, die im Übrigen zu keinem persönlichen Gespräch bereit war, eingegangen werden. Für das Areal, in dem die Nachverdichtung stattfindet, wurde der Phantasiename Jaminpark erfunden und in der Werbung ein Wohnen im Park versprochen. Der Name suggeriert weite Grünanlagen, wird aber von den Bewohner_innen angesichts der vielen Baumfällungen als 
Hohn empfunden. Es mussten über 1.0oo Bäume gefällt werden (Erlanger Nachrichten 10.02.2018). Dem Quartier war einstmals eine Einzigartigkeit bezüglich der Flora und Fauna zugesprochen worden. „Für ein bebautes Gebiet ist sie sogar einmalig! [...] Teilweise fand man hier auch bedrohte Tierarten.“ (Interview Bewohner_innen Rathenau 2, 29.06.2018) Vertreter_innen der Stadtplanung verteidigen diese Maßnahmen als unumgänglich und ökologisch verträglich mit dem Hinweis auf Ausgleichsmaßnahmen, insbesondere die Pflanzung neuer Bäume.

Bezüglich der Sozialverträglichkeit der Maßnahmen äußerte die GBW: „Wir planen eine sozialverträgliche Modernisierung. U. a. heißt das: Keiner der betroffenen Mieter wird sein gewohntes Wohnumfeld verlassen müssen, weil er/sie sich eine Modernisierungsumlage nicht leisten kann." (GBW o. J.b) Erfahrungsgemäß aber erwarten die Bewohner_innen des Quartiers durchaus Mieterhöhungen, denn nach energetischer Sanierung können 2,60 Euro pro Quadratmeter mehr verlangt werden, wodurch manche Mieter_innen mit 6o Prozent höheren Mieten rechnen müssten (Interview GBW Mietergemeinschaft, 27.06.2018; Interview Bewohner_innen Rathenau 2, 29.06.2018). Schon früher habe es Mieterhöhungen der GBW gegeben, welche aber teilweise zurückgenommen werden mussten, als Bewohner_innen diese zur Kontrolle an den Deutschen Mieterbund weitergaben. Die GBW habe sich dann auf Systemfehler berufen, diese angeblichen Fehler aber keineswegs für alle Mieter_innen korrigiert. „Die GBW vertritt die Meinung, dass Mieterhöhungen nur zurückgenommen werden müssen, wenn sich die Mieter beschweren und Hilfe suchen." (Interview GBW Mietergemeinschaft, 27.06.2018)

Die GBW-Gruppe scheint intensiv an einer Steigerung der Mieteinnahmen interessiert zu sein. In diesem Zusammenhang ist es naheliegend, dass die entstehende Mikrosegregation durchaus erwünscht ist, um in den privilegierteren Lagen auch höhere Mieten verlangen zu können. Im Folgenden, zweiten Beispiel wird zudem deutlich, dass sich bei gezielter Neubebauung eines kompletten Quartiers umweltbezogene Mikrosegregation noch deutlich differenzierter anhand der Gebäudekubatur ablesen lässt.

\section{Schaffung privilegierter Wohnlagen im Röthelheimpark}

Das zweite Untersuchungsgebiet liegt im Erlanger Stadtteil Röthelheimpark und befindet sich im Osten der Stadt Erlangen. Der Stadtteil wurde von 1997 bis 2014 entwickelt, wobei der von uns betrachtete Raumausschnitt komplett neu als Wohngebiet entwickelt wurde.

\subsection{Planungsprozess Röthelheimpark}

Bevor 1997 mit den Planungen für einen neuen Stadtteil begonnen wurde, befand sich auf dem 151 Hektar großen Gelände ein US-amerikanischer Militärstützpunkt, der 1993 aufgegeben wurde. Im Jahr 1994 erwarb die Stadt Erlangen das Gebiet und wies zunächst ein 25 Hektar großes Naturschutzgebiet aus. Für den restlichen Teil des Grundstückes lobte die Stadt einen städtebaulichen Wettbewerb aus, den 1995 ein Münchner Planungsbüro gewann. Mit Baubeginn 1997 war es das Ziel, ein „urbanes innenstadtnahes Wohnen 
in familien- und kinderfreundlichem Umfeld mit kurzen Wegen zu Grünbereichen, Infrastrukturen und Arbeitsplätzen“ zu ermöglichen (Stadt Erlangen 2011: 30). Konzepte wie „Stadt der kurzen Wege“, „kompakte Stadt“ (Nutzungsmischung aus Wohnen, Gemeinbedarfseinrichtungen, Gewerbe, Büros, Dienstleistungen, Handel und Einrichtungen der Universität) und „autofreie Stadt“ (Tiefgaragen) sollten realisiert werden (Stadt Erlangen 2011: $30 \mathrm{ff}$.).

Der ursprüngliche Entwurf sah Wohnraum für rund 9.00o Einwohner_innen vor, wurde jedoch im Planungsprozess deutlich auf zunächst 6.00o und später auf 3.500 reduziert (Stadt Erlangen 2001). Dabei blieb die Grundstruktur des Planes erhalten, jedoch wurde die Bebauungshöhe der Gebäude reduziert, im Nord-Westen wurde der Siemens AG ein Areal zur Verfügung gestellt und im Südosten - dies ist das von uns untersuchte Gebiet - wurden große Teile des geplanten Geschosswohnungsbaus durch Reihen- beziehungsweise Einfamilienhäuser, einen Baumarkt und ein Bekleidungsgeschäft ersetzt.

\section{2. „Bauen nach Investorenlage“}

Die Reduktion des Wohnraums sei aus Sicht der Vertreter_innen der Stadtplanung beziehungsweise der mit der Koordinierung beauftragten Projektgruppe Röthelheimpark alternativlos gewesen. Es hätte von Seiten der Investor_innen kaum Interesse gegeben, in Geschosswohnungsbau zu investieren, „weder in Eigentumswohnungen, schon gar nicht erst in Mietwohnungen“ (Interview Mitarbeiter_in der Stadtplanung 2, 19.04.2018). Es habe auch keinen Bedarf an neuem Wohnraum für 9.00o Einwohner_innen in fast ausschließlich Geschosswohnungsbau gegeben (Interview Mitglied Projektgruppe Röthelheimpark: 28.04.2018). Ein Mitglied des Stadtrates beteuert hingegen, der Wohnraum sei keinesfalls aufgrund fehlender Nachfrage reduziert worden und bezichtigt den damaligen Stadtrat „Bauen nach Investorenlage“ betrieben zu haben. Die Stadt sei „grundsätzlich zurückgewichen, wenn ein Investor etwas Anderes wollte. Dann wurden auch keine allzu großen Diskussionen geführt.“(Interview Mitglied Stadtrat, 26.06.2018) Der Stadtrat habe sich nicht an den Bedürfnissen der Bewohner_innen, sondern an denen der Investor_innen orientiert. Der Bedarf an Wohnungen sei durchaus hoch gewesen, denn es habe seit dem Ende der 1970er Jahre noch nie genügend Wohnungen in Erlangen gegeben.

Diese Aussage bestätigt sich in einem Bericht des Amts für Statistik der Stadt Erlangen (1990: 1). Dieser weist für die späten 1980er Jahre eine mangelnde Wohnraumentwicklung aus und prognostiziert für die darauffolgenden Jahre eine zu erwartende Zunahme des Arbeitsplatzangebotes mit entsprechendem, damit einhergehendem Wohnungsbedarf. Dass bei der damaligen Wohnungspolitik aber nur „ein Teil der zusätzlich Beschäftigten mit ihren Familien eine Wohnung im Stadtgebiet beziehen können“, schien bei den "geplanten Wohnbauformen (Einfamilien- oder Mehrfamilienhäuser)" schon absehbar gewesen zu sein. Tatsächlich wies Erlangen schon lange vor den anderen Großstädten der Umgebung (Nürnberg, Fürth) sehr hohe Mietpreise auf. Es kann also davon ausgegangen werden, dass die Nachfrage nach mehr Wohnraum auch im Röthelheimpark durchaus vorhanden gewesen wäre. 


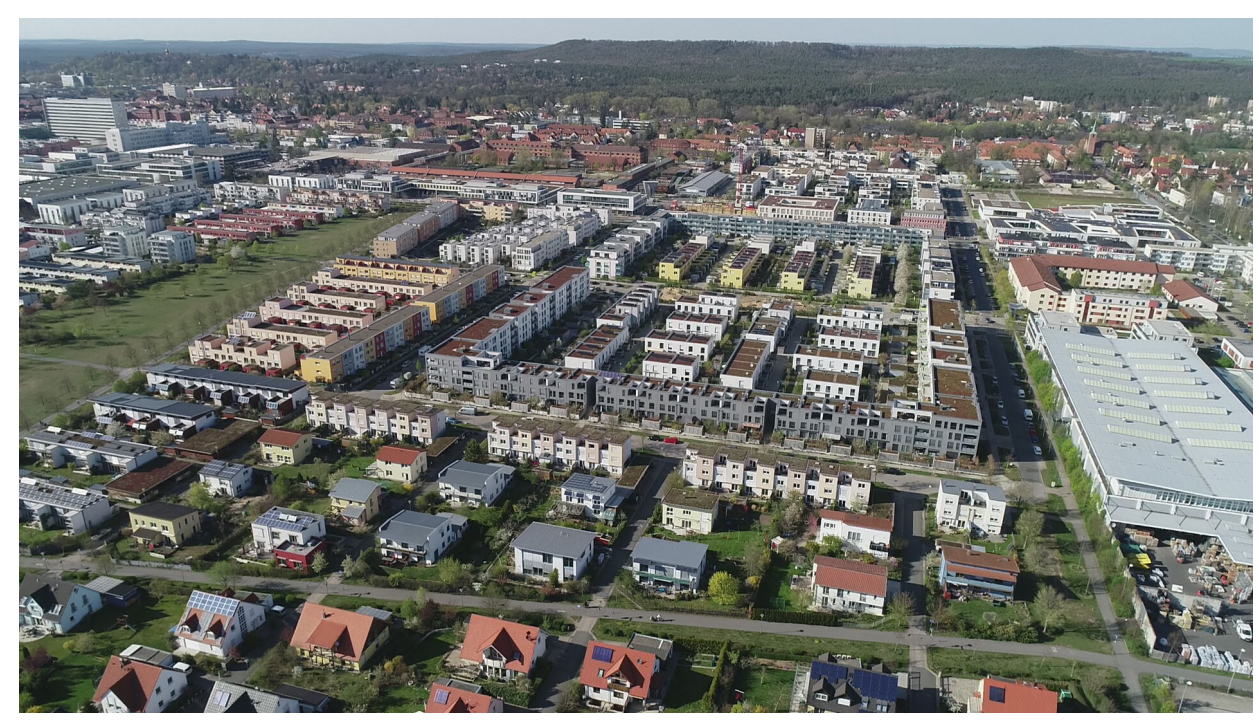

Unsere Interviewpartnerin betont, dass der Wohnraum nicht zuletzt verknappt worden sei, um den Stadtteil für eine zahlungskräftige Klientel attraktiv zu machen (Interview Mitglied Stadtrat, 26.06.2018). Als kreisfreie Stadt verfügt Erlangen nur über ein sehr begrenztes Stadtgebiet. Es habe die Sorge bestanden, dass die wohlhabende Bevölkerung aufs Land zieht, die Pendlerströme zunehmen und die Einkommenssteuer in die Umlandkommunen abfließt. Die ,Leistungsträger_innen“ sollten in der Stadt gehalten werden. In dieses Bild passt nicht zuletzt auch die ortsnahe Ansiedlung der Franconian International School, einer exklusiven Privatschule mit Eliteanspruch (Abbildung 4). Von Seiten der Planung wurde auch keineswegs bestritten, dass soziale Mischung hier gar nicht angestrebt wurde. Es sei darüber nachzudenken, „ob ich jetzt dauernd in jeder Ecke der Stadt irgendwie die Durchmischung haben will?" (Interview Mitglied Projektgruppe Röthelheimpark, 28.04.2018). Man könne in Erlangen auch überhaupt nicht von sozialen Brennpunkten sprechen. Zwar gäbe es segregierte Bereiche und es sei „schon die ärmere Bevölkerung, die da wohnt. Bloß es ist alles verhältnismäßig klein von der Größenordnung und Fläche.“ (ebd.) Im Vergleich mit sozialen Brennpunkten in Städten wie Berlin - „wo sich dann wirklich alles sozial Schwächere ballt“ (ebd.) - sei das zu vernachlässigen. Als interessante Randnotiz erscheint in diesem Zusammenhang die Tatsache, dass Erlangen von einer aktuellen Studie als die am stärksten segregierte westdeutsche Stadt ausgewiesen wird (Helbig/Jähnen 2018: 30).

\subsection{Verteilung umweltbedingter Belastungen}

In diesem bewusst für eine zahlungskräftige Klientel entworfenen Quartier lässt sich nun eine differenzierte, graduelle Verteilung der umweltbedingten Belastungen und Ressourcen nach finanzieller Leistungsfähigkeit erkennen (Abbildung 3 u. 4). Insbesondere die Belastungen durch den Straßenverkehr wurden im Röthelheimpark gezielt verteilt. Die Allee am Röthelheimpark dient als einzige Durchfahrtsstraße durch den Stadtteil und ist entsprechend stark befahren. Entlang dieser Allee und der nach Osten hin das untersuchte Quartier begrenzenden Kurt-Schumacher-Straße kommt es aufgrund von Pendlerströmen zu erheblichen Verkehrsbelastungen. Dort, wo sich diese beiden Straßen kreuzen und eine Ampelanlage zu Stauungen und
Abb. 3 Luftbild des untersuchten Quartiers im Röthelheimpark (Foto: Jan Gemeinholzer) 
entsprechenden Lärm- und Abgasbelastungen führt, steht ein L-förmiger Bau mit geförderten Wohnungen, der effektiv die Belastung dieser Kreuzungen für die dahinter liegenden Bauten mindert (Abbildung 4: Signatur D). Ebenso wurden entlang der Allee am Röthelheimpark mehrgeschossige Bauten errichtet, die das südlich gelegene Quartier von Lärm und Abgasen abschirmen (Abbildung 4: Signatur B). Um die verkehrsbedingten Lärmbelastungen zu reduzieren, wurden hier nach Angaben der Stadtplanung die Wohnräume von der Hauptstraße weg nach Süden orientiert (Interview Mitarbeiter_in Stadtplanung, 19.04.2018). Von den Anwohner_innen selbst wird die schalldämmende Wirkung dieser Grundrissorientierung jedoch nur bedingt bestätigt (Gruppeninterview Bewohner_innen Röthelheimpark, 26.06.2018).

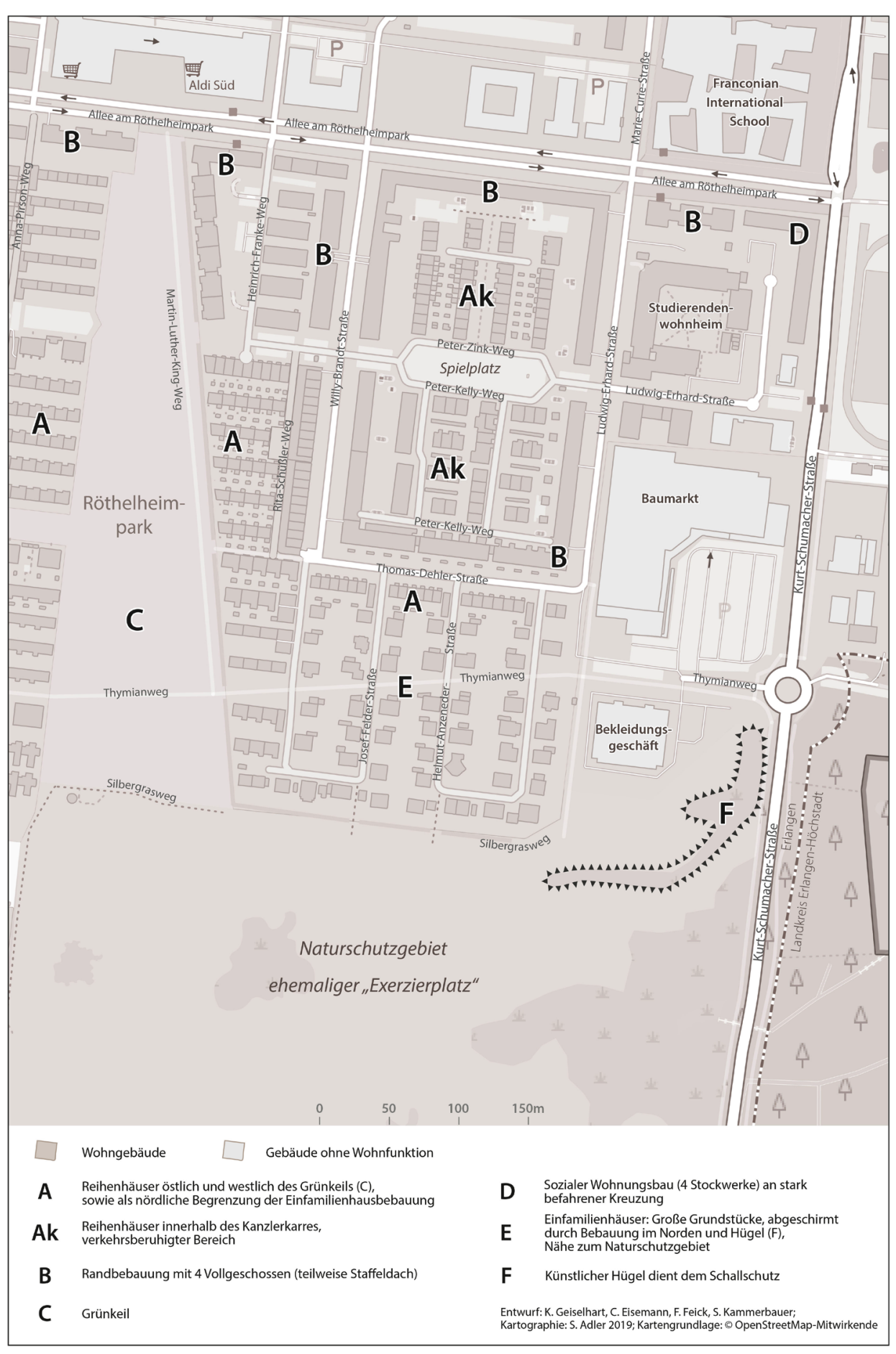

Abb. 4 Wohngebäudestruktur eines Quartiers im Röthelheimpark (Quelle: Eigene Darstellung, Kartographie: Stephan Adler) 
Diese Bebauung mit Geschosswohnungen wurde entlang der Straßen fortgesetzt, die den Zugangsverkehr des Quartiers aufnehmen (Abbildung 4: Signatur B). Die Willy-Brand-Straßeim Westen und die Ludwig-Erhard-Straße im Osten begrenzen das sogenannte ,Kanzlerkarree', welches durch diese randständige Bebauung kaum Umweltbelastungen durch Verkehr ausgesetzt ist (Abbildung 3). „Die atmen die Abgase für uns weg“ (Gruppeninterview Bewohner_innen Röthelheimpark, 26.06.2018). So stellt das Kanzlerkarree eine künstlich geschaffene Nische dar, die einen privilegierten Bereich für Reihenhäuser und exklusive moderne Stadteigenheimarchitektur schafft (Abbildung 4: Signatur Ak).

\subsection{Verteilung von Umweltressourcen}

Eine Besonderheit des Quartiers ist die Anbindung an eine weiträumige Grünachse in Nord-Süd-Richtung. In Form eines Keils durchzieht sie den Stadtteil Röthelheimpark (Abbildung 4: Signatur C). An der Ostseite wird dieser Grünkeil begleitet von einer kleinen Allee, die im Norden direkt auf den Georg-Marshall-Platz zuläuft, den zentralen Campus der gesamten Anlage des Stadtteils. Im Süden setzt sich der Grünkeil in ein Naturschutzgebiet fort, wodurch eine Grünachse entsteht, die den Anwohner_innen Möglichkeiten zur Naherholung bietet. Sie dient auch als Frischluftschneise und wird von den Bewohner_innen als wohltuend empfunden (Gruppeninterview Bewohner_innen Röthelheimpark, 26.06.2018).

Westlich und östlich dieses Grünkeils befinden sich weitere Reihenhauszeilen (Abbildung 4: Signatur A). Die Lagen dieser Reihenhäuser sind dadurch gekennzeichnet, dass hier durchaus mit gewissen Belastungen durch Freizeitaktivitäten zu rechnen ist. Speziell der im Grünkeil in NordSüd-Richtung verlaufende Fuß- und Radweg stellt eine Quelle möglicher Beeinträchtigungen dar. Es kann von einem Zustrom der Nutzung von Norden her ausgegangen werden. Im Plan lässt sich gut erkennen, wie dieser Weg anfangs von Geschoßwohnungen und später von Reihenhäusern flankiert wird, deren Grundstücksgrößen mit abnehmender Belastung nach Süden hin zunehmen. Dies kann als Indikator für die zunehmende Exklusivität der Wohnlage gelesen werden.

Deutlich privilegiert aber ist die Lage der Einfamilienhäuser südlich des Kanzlerkarrees (Abbildung 4: Signatur E). Schon bei der Planung muss klar gewesen sein, dass diese Wohnlagen bei diesen Grundstücksgrößen, den Erlanger Grundstückspreisen und der vorgesehenen Bebauungsstruktur nur besonders betuchten Personen vorbehalten sein würden. Dieser Bereich wird im Norden durch eine zusätzliche Zeile aus Reihenhäusern (A) vom Zufahrtsverkehr in der Thomas-Dehler-Straße und nach Osten hin durch einen künstlich zu diesem Zweck aufgeschüttetem Hügel (F) von den Verkehrsbelastungen der Kurt-Schumacher-Straße abgeschirmt. Selbige Funktion übernehmen der Baumarkt und das Bekleidungsgeschäft. Von Süden her ist hingegen kaum mit Belastung zu rechnen. Hier befindet sich angrenzend das schon erwähnte Naturschutzgebiet. Außerdem wurde hier ein Streifen mit altem Baumbestand naturnah belassen - es drängt sich der Eindruck auf, dass die südlichsten Grundstücke zusätzlich vor neugierigen Blicken geschützt werden sollten. 


\section{Fazit}

Die vorliegenden Beispiele zeigen, dass sich Umweltbelastungen und Umweltressourcen ungleichmäßig auf die Bewohner_innen der untersuchten Nachbarschaften verteilen. Sie werden durch die Planung der Gebäudestrukturen graduell entsprechend der finanziellen Leistungsfähigkeit der Bewohner_innen verteilt: Dort, wo zwar eine Nähe zu Grünanlagen gegeben ist, aber mit vielen Spaziergänger_innen oder auch Lärm durch Freizeitaktivitäten zu rechnen ist, wird eine weniger exklusive Bebauung auf kleineren Grundstücken geplant als dort, wo Wohnlagen an eher ruhigen, naturnahen Flächen liegen und wirkungsvoll gegen belastende Umwelteinflüsse abgeschirmt sind. Der städtischen Auflage, Sozialwohnungen zu schaffen, um soziale Mischung zu gewährleisten, wird nachgekommen, indem geförderte Wohneinheiten in den durch Lärm und Abgase am meisten belasteten Lagen errichtet werden. Dadurch wird eine gegen Umweltbelastungen abschirmende Funktion für die dahinter liegenden Wohneinheiten erreicht. Die poor doors liegen in Erlangen an viel befahrenen Kreuzungen und Straßen.

Unsystematische Beobachtungen der Autor_innen legen nahe, dass derartige umweltbezogene Mikrosegregation auch anderenorts zu beobachten ist, doch steht eine methodisch strukturierte Abschätzung der Reichweite dieses Phänomens noch aus. Insbesondere wäre es interessant, die Situation in angloamerikanischen Städten zu betrachten, wo Umweltfaktoren in der Debatte um poor doors bisher keine Rolle spielen.

Die hier beschriebene Form der umweltbezogenen Mikrosegregation findet innerhalb der Quartiere auf Gebäudezeilenebene statt und ist damit so kleinräumig, dass sie sich nicht mittels gängiger Sozialraumanalysen erschließt. Sie produziert bei steigender Ungleichheit statistisch nivellierende Effekte, denn in Gebieten mit statushohen Bewohner_innen drückt sie die Kennzahlen, während die statusniederen Gebiete (statistisch) angehoben werden. Selbst neuere Konzepte des Umweltgerechtigkeitsmonitoring, wie etwa die Restrukturierung räumlicher Statistik mittels lebensweltlich orientierter Räume (LOR) (Bömermann/Jahn/Nelius 2006), können diese Mikrosegregation nur unzureichend erfassen. Dieser Unsichtbarkeit könnte nur mit der Entwicklung angemessener Erhebungsinstrumente begegnet werden.

Michael Flitner (2003) zufolge sollte Umweltgerechtigkeit nicht allein unter dem Aspekt der Verteilungsgerechtigkeit behandelt werden: Wenn nur gefragt werde, wer wie vielen Belastungen ausgesetzt sei und wer auf wie viele Ressourcen zurückgreifen könne, dann gerieten die eigentlich politischen Fragen von Anerkennungsgerechtigkeit aus dem Blick. In dieser auf Axel Honneths (1994) Begriff gesellschaftlicher Anerkennung Bezug nehmenden Perspektive geht es um Fragen der kulturellen Repräsentation bestimmter Bevölkerungsgruppen, also um die Frage danach, auf welcher Vorstellungsund Wertebasis umweltbezogene Ungleichverteilungen hergestellt werden. In vorliegendem Fall zeigt sich, dass das Recht, sich gute Lebensbedingungen zu erkaufen, ebenso wenig in Frage gestellt wird wie die Rechtmäßigkeit der Investor_inneninteressen. Die interviewten Protagonist_innen der betrachteten Stadtentwicklungsprojekte standen offen zu der ungleichen 
Verteilung von Umweltressourcen und Umweltbelastungen. Sie waren damit implizit der Auffassung, dass man denjenigen, die nicht über die notwendigen finanziellen Mittel verfügen, und denjenigen, die durch Sozialleistungen von der öffentlichen Hand unterstützt werden, durchaus mehr Umweltbelastungen zumuten könne. Beachtet man aber, dass mit diesen Belastungen tatsächlich handfeste gesundheitliche Gefahren verbunden sind, dann erscheinen die Verhältnisse als ernst zu nehmende Diskriminierung. Offensichtlich wird es als gerechtfertigt empfunden, dass ärmere Bevölkerungsgruppen mehr Gesundheitsrisiken in Kauf nehmen müssen als wohlhabende.

Die einkommensorientierten Ungleichheiten zeichneten sich schon in den städtebaulichen Planungen differenziert ab. Zudem wurden im Zuge der Planungs- und Baumaßnahmen ursprünglich freiwillige Zugeständnisse und städtische Beschränkungen bezüglich des Naturschutzes sowie der sozialen Nachhaltigkeit sukzessive aufgeweicht. Umweltbedingungen wurden gezielt nach finanzieller Leistungsfähigkeit verteilt. Die Beispiele haben gezeigt, wie Euphemismen (,Wohnen im Park', ,Baumersatz') eingesetzt werden, um die Planungen in einem besseren Licht erscheinen zu lassen. Sobald einmal bestimmte Hürden genommen sind (Bürgerbeteiligungsverfahren, wichtige Stadtratsbeschlüsse etc.), wird im Laufe der Planungen und Umsetzungen auch von den einstmals kommunizierten Strategien und Zielen abgewichen (Zahl der Wohneinheiten, Geschosswohnungsbau ersetzt durch Einfamilienhäuser). Um zu vermeiden, dass der Anspruch auf Umweltgerechtigkeit derart klammheimlich in den Wirren der Planungs- und Umsetzungsprozesse versandet, halten Böhme, Dilger und Quilling (2018) ein integriertes Verwaltungshandeln für notwendig. Darunter verstehen sie ein ressortübergreifendes, gemeinsames Handeln aller raumplanerischen, sozialen, wirtschaftlichen sowie gesundheitsfördernden Verwaltungsbereiche einer Stadt. Zudem sei ein angemessenes Monitoring zur Kontrolle der Wirksamkeit der getroffenen Maßnahmen unumgänglich. Umweltgerechtigkeit muss ihres Erachtens fest im politischen Willen und den Verwaltungsstrukturen verankert werden. Darüber hinaus bedarf es des aufmerksamen kritischen Blickes einer gesellschaftlichen Öffentlichkeit.

Dieser Artikel wurde durch den Open Access Publikationsfonds der Friedrich-Alexander-Universität Erlangen-Nürnberg gefördert. 


\section{Autor_innen}

Klaus Geiselhart ist Geograph mit den Arbeitsschwerpunkten Geographische Gesundheitsforschung und Urban Studies.

klaus.geiselhart@fau.de

Carolin Eisemann ist Kulturgeographin und Angestellte in einem Ingenieurbüro mit den Arbeitsschwerpunkten Stadtentwicklung und Stadtplanung.

c.eisemann@gmx.de

Fabian Feick ist Kulturgeograph mit dem Schwerpunkt Stadtentwicklung und Stadtplanung. fabian.feick@fau.de

Stefan Kammerbauer ist Kulturgeograph mit dem Schwerpunkt Stadtentwicklung und Stadtforschung.

stefan.kammerbauer@freenet.de

\section{Literatur}

Aehnelt, Reinhard (2011): Trends und Ausmaß der Polarisierung in deutschen Städten. In: Walter Hanesch (Hg.), Die Zukunft der „Sozialen Stadt“. Strategien gegen soziale Spaltung und Armut in den Kommunen. Wiesbaden: Springer, 63-79.

Bacqué, Marie-Hèléne / Fijalkow, Yankel (2012): Social mix as the aim of controlled gentrification process: the example of the Goutte d'Or district in Paris. In: Gary Bridge / Tim Butler / Loretta Lees (Hg.), Mixed Communities - Gentrification by stealth? Bristol: The Policy Press, 115-132.

Barton, Hugh / Grant, Marcus (2006): A health map for the local human habitat. In: The Journal for the Royal Society for the Promotion of Health 126/6, 252-253.

Belina, Bernd / Gestring, Norbert / Müller, Wolfgang / Sträter, Detlev (2011): Einleitung. In: Bernd Belina / Norbert Gestring / Wolfgang Müller / Detlev Sträter (Hg.), Urbane Differenzen - Disparitäten innerhalb und zwischen Städten. Münster: Westfälisches Dampfboot, 7-13.

Böhme, Christa / Bunge, Christiane / Preuß, Thomas (2016): Umweltgerechtigkeit in der Stadt - zur integrierten Betrachtung von Umwelt, Gesundheit, Sozialem und Stadtentwicklung in der kommunalen Praxis. In: Umweltpsychologie 20/2, 137-157.

Böhme, Christa / Dilger, Ulrich / Quilling, Eike (2018): Integriertes Verwaltungshandeln für eine gesundheitsfördernde Stadtentwicklung. In: Sabine Baumgart / Heike Köckler / Anne Ritzinger / Andrea Rüdiger (Hg.), Planung für gesundheitsfördernde Städte. Hannover: ARL, 135-144.

Böhme, Christa / Preuß, Thomas / Bunzel, Arno / Reimann, Bettina / Seidel-Schulze, Antje / Landua, Detlef (2005): Umweltgerechtigkeit im städtischen Raum - Entwicklung von praxistauglichen Strategien und Maßnahmen zur Minderung sozial ungleich verteilter Umweltbelastungen. http://www.umweltbundesamt.de/publikationen/ umweltgerechtigkeit-im-staedtischen-raum (letzter Zugriff am 31.03.2019).

Bömermann, Hartmut / Jahn, Susanne / Nelius, Kurt (2006): Lebensweltlich orientierte Räume im Regionalen Bezugssystem (Teil 1). Werkstattbericht zum Projekt „Vereinheitlichung von Planungsräumen“. In: Berliner Statistik Monatsschrift 8/6, 366-372. https:// www.stadtentwicklung.berlin.de/planen/basisdaten_stadtentwicklung/lor/download/ BerlinerStatistik0608.pdf (letzter Zugriff am 31.03.2019).

Bolte, Gabriele / Bunge, Christiane./ Hornbeck, Claudia / Köckler, Heike / Mielck, Andreas (2012): Umweltgerechtigkeit durch Chancengleichheit bei Umwelt und Gesundheit. Eine Einführung in die Thematik und Zielsetzung dieses Buches. In: Gabriele Bolte / Christiane Bunge / Claudia Hornbeck / Heike Köckler / Andreas Mielck (Hg.), Umweltgerechtigkeit. Chancengleichheit bei Umwelt und Gesundheit - Konzepte, Datenlage und Handlungsperspektiven. Bern: Huber, 15-38.

Brailich, Adam / Germes, Mélina / Schirmel, Henning / Glasze, Georg / Pütz, Robert (2008): Die diskursive Konstitution von Großwohnsiedlungen in Deutschland, Frankreich und Polen. In: Europa Regional 16/3, 113-128. 
Brake, Klaus (2011): „Reurbanisierung“ - janusköpfiger Paradigmenwechsel, wissensintensive Ökonomie und neuartige Inwertsetzung städtischer Strukturen. In: Bernd Belina / Norbert Gestring / Wolfgang Müller / Detlev Sträter (Hg.), Urbane Differenzen Disparitäten innerhalb und zwischen Städten. Münster: Westfälisches Dampfboot, 69-96.

Bürkner, Hansjoachim (2011): Sozialräumliche Disparitäten und soziale Mischung. Aktuelle Diskurslinien in Forschung und gesellschaftlicher Praxis. In: Bernd Belina / Norbert Gestring / Wolfgang Müller / Detlev Sträter (Hg.), Urbane Differenzen - Disparitäten innerhalb und zwischen Städten. Münster: Westfälisches Dampfboot, 16-42.

Cheshire, Paul (2012): Social mix and urban policy. In: Gary Bridge / Tim Butler / Loretta Lees (Hg.), Mixed Communities - Gentrification by stealth? Bristol: The Policy Press, $17-33$.

Claßen, Thomas / Heiler, Angela / Brei, Björn (2012): Urbane Grünräume und gesundheitliche Chancengleichheit - längst nicht alles im „grünen Bereich“. In: Gabriele Bolte / Christiane Bunge / Claudia Hornbeck / Heike Köckler / Andreas Mielck (Hg.), Umweltgerechtigkeit. Chancengleichheit bei Umwelt und Gesundheit - Konzepte, Datenlage und Handlungsperspektiven. Bern: Huber, 113-123.

Claßen, Thomas / Mekel, Odile (2016): Fachplan Gesundheit - ein neues Konzept für eine nachhaltige, gesundheitsförderliche Kommunalentwicklung. In: Public Health Forum 24/4, 275-277.

Dahlgren, Göran / Whitehead, Margaret (1992): Policies and strategies to promote equity in health. http://whqlibdoc.who.int/euro/-1993/EUR_ICP_RPD414\%282\%29.pdf (letzter Zugriff am 06.05.2017).

Dangschat, Jens S. (2017): Armut und Stadterneuerung - zwei Seiten einer Medaille? In: Uwe Altrock / Ronald Kunze (Hg.), Stadterneuerung und Armut. Jahrbuch Stadterneuerung 2016. Wiesbaden: Springer, 13-35.

Erlanger Nachrichten (2018): Streit über Nachverdichtung: Bäume fallen in Erlangen. http://www.nordbayern.de/region/erlangen/streit-uber-nachverdichtung-baumefallen-in-erlangen-1.7214908?rssPage=RXJsYW5nZW4 (letzter Zugriff am 31.03.2019).

Farwick, Andreas (2012): Segregation. In: Frank Eckhardt (Hg.), Handbuch Stadtsoziologie. Wiesbaden: Springer VS, 381-420.

Faßmann, Heinz / Franz, Yvonne (2015): Soziale Mischung und soziale Durchmischung. Ein gesellschaftspolitisches Ideal zwischen Anspruch und Wirklichkeit. In: Judith Fritz / Nino Tomaschek (Hg.), Die Stadt der Zukunft. Aktuelle Trends und zukünftige Herausforderungen. Münster u. a.: Waxmann, 193-207.

Flitner, Michael (2003): Umweltgerechtigkeit. Ein neuer Ansatz der sozialwissenschaftlichen Umweltforschung. In: Peter Meusburger / Thomas Schwan (Hg.), Humanökologie. Ansätze zur Überwindung der Natur-Kultur-Dichotomie. Stuttgart: Steiner, 139-160.

GBW (2018): Quartier Jaminpark. Mieterinformationsveranstaltung am 24.04.2018. https://www.gbw-gruppe.de/pdf/quartier-erlangen/180424_mieterveranstaltungerlangen.pdf (letzter Zugriff am 21.11.2018).

GBW (o. J.a): Quartier Erlangen - Das Projekt. https://www.gbw-gruppe.de/projekte/ erlangen/quartier-jaminpark/projekt (letzter Zugriff am 21.11.2018).

GBW (o. J.b): Unser Quartier hat einen neuen Namen. https://www.gbw-gruppe.de/ projekte/erlangen/quartier-jaminpar (letzter Zugriff am 21.11.2018).

Goebel, Jan / Hoppe, Lukas (2015): Ausmaß und Trends sozialräumlicher Segregation in Deutschland. In: Lebenslagen in Deutschland. Armuts- und Reichtumsberichterstattung der Bundesregierung. Berlin.

Häußermann, Hartmut (2012): Wohnen und Quartier: Ursachen sozialräumlicher Segregation. In: Ernst-Ulrich Huster / Jürgen Boeckh / Hildegard Mogge-Grotjahn (Hg.), Handbuch Armut und Soziale Ausgrenzung. Wiesbaden: Springer VS, 336-349.

Harlander, Tilman (2012): Einmischung - wie deutsche Städte die soziale Mischung fördern. In: Stadtbauwelt 196/48, 51-61.

Harlander, Tilman / Kuhn, Gerd (2012): Segregation und Mischung in Europa. In: Stadtbauwelt 196/48, 17-27.

Helbig, Marcel / Jähnen, Stephanie (2018): Wie brüchig ist die soziale Architektur unserer Städte? Trends und Analysen der Segregation in 74 deutschen Städten. https://bibliothek. wzb.eu/pdf/2018/p18-o01.pdf (letzter Zugriff am 31.03.2019).

Helbrecht, Ilse (2009): „Stadt der Enklaven“? Neue Herausforderungen für Städte in der globalen Wissensgesellschaft. In: Neues Archiv für Niedersachsen 2, 2-17.

Helbrecht, Ilse (2016): Gentrification und Verdrängung. In: Ilse Helbrecht (Hg.), Gentrifizierung in Berlin. Verdrängungsprozesse und Bleibestrategien. Bielefeld: transcript, 9-16. 
Honneth, Axel (1994): Kampf um Anerkennung. Zur moralischen Grammatik sozialer Konflikte. Frankfurt am Main: Suhrkamp.

Kabisch, Sigrun / Ueberham, Maximilian / Söding, Max (2018): Dynamiken der Großwohnsiedlung Leipzig-Grünau aus Bewohnersicht in der Langzeitperspektive. In: Uwe Altrock / Nico Grunze / Sigrun Kabisch (Hg.), Großwohnsiedlungen im Haltbarkeitscheck. Differenzierte Perspektiven ostdeutscher Großwohnsiedlungen. Wiesbaden: Springer, 119-143.

Kistemann, Thomas / Völker, Sebastian / Lengen, Charis (2010): Stadtblau - Die gesundheitliche Bedeutung von Gewässern im urbanen Raum. In: NUA (Natur- und Umweltschutz Akademie des Landes Nordrhein-Westfalen) (Hg.), Die Bedeutung von Stadtgrün für die Gesundheit. Recklinghausen: NUA, 61-66.

Klimeczek, Heinz-Josef (2014): Umweltgerechtigkeit im Land Berlin - Zur methodischen Entwicklung des zweistufigen Berliner Umweltgerechtigkeitsmonitorings. In: UMID (Umwelt und Mensch - Informationsdienst) 2, 16-22. https://www.umweltbundesamt. de/sites/default/files/medien/378/publikationen/umweltgerechtigkeit_im_land_ berlin_16-22.pdf (letzter Zugriff am 31.03.2019).

Kowarik, Ingo / Bartz, Robert / Brenck, Miriam (2016): Ökosystemleistungen in der Stadt Gesundheit schützen und Lebensqualität erhöhen. Berlin, Leipzig: Technische Universität Berlin, Helmholtz-Zentrum für Umweltforschung - UFZ.

Kronauer, Martin (2002): Die neue soziale Frage: Armut und Ausgrenzung in der Großstadt heute. In: Uwe-Jens Walther (Hg.), Soziale Stadt - Zwischenbilanzen. Ein Programm auf dem Weg zur Sozialen Stadt? Wiesbaden: VS, 45-55.

Lees, Loretta (2008): Gentrification and Social Mixing: Towards an Inclusive Urban Renaissance? In: Urban Studies 45/12, 2449-2470

Licea, Melkorka (2016): 'Poor door' tenants of luxury tower reveal the financial apartheid within. In: New York Post, 17.01.2016. https://nypost.com/2016/01/17/poor-doortenants-reveal-luxury-towers-financial-apartheid/ (letzter Zugriff am 31.03.2019).

von Lojewski, Hilmar (2013): Zum Verhältnis von sozialer Durchmischung, Segregation und Gentrifizierung. In: vhw FWS 4, 175-179.

LZG.NRW (Landeszentrum Gesundheit Nordrhein-Westfalen) (2016): Leitfaden Gesunde Stadt. Hinweise für Stellungnahmen zur Stadtentwicklung aus dem Öffentlichen Gesundheitsdienst. https://www.lzg.nrw.de/_media/pdf/service/Pub/2016_druckfrisch/ lzg-nrw_leitfaden_gesunde_stadt_2016.pdf (letzter Zugriff am 06.05.2017).

Massey, Douglas S. / Denton, Nancy A. (1993): American Apartheid. Segregation and the Making of the Underclass. Cambridge: Harvard University Press.

Mayring, Philipp (2015): Qualitative Inhaltsanalyse. Grundlagen und Techniken. Weinheim/ Basel: Beltz.

Meubrink, Yuca (2016): Segregation durch Architektur als Produkt Londoner Wohnungspolitik. In: Frank Othengrafen / Brigitta Schmidt-Lauber / Christine Hannemann / Jörg Pohlan / Frank Roost (Hg.), Jahrbuch StadtRegion: Planbarkeiten. Herausforderungen und Dynamiken der räumlichen Planung. Opladen: Barbara Budrich, 152-166.

Millian, Peter (2009): Siemens-Wohnungen haben einen neuen Vermieter. http://www. nordbayern.de/region/erlangen/siemens-wohnungen-haben-neue-vermieter-1.661992 (letzter Zugriff am 31.03.2019).

NYU Furman Center (2015): Discussion 12: The Poor Door Debate. https://furmancenter. org/research/iri/discussions/the-poor-door-debate (letzter Zugriff: 31.07.2019).

Pitzke, Mark (2014): Luxusbauten in New York. Arme müssen durch die Hintertür. In: Spiegel Online, 30.10.2014. http://www.spiegel.de/forum/wirtschaft/luxusbauten-newyork-arme-muessen-durch-die-hintertuer-thread-178774-1.html (letzter Zugriff am 31.03.2019).

Stadt Erlangen (1990): Statistik aktuell: Gesamtprognose Erlangen 1990-2005. https:// www.erlangen.de/Portaldata/1/Resources/o8o_stadtverwaltung/dokumente/ statistik/30S_B_MB_1990_12-11.pdf (letzter Zugriff am 13.08.2019).

Stadt Erlangen (2011): Der Röthelheimpark. Vom Militärgelände zum Vorzeigestadtteil eine Erfolgsgeschichte. https://www.erlangen.de/Portaldata/1/Resources/o30_leben_ in_er/dokumente/roethelheimpark/RHP_DerRoethelheimpark-2011.pdf (letzter Zugriff am 30.03.2019).

Stadt Erlangen (2018): Bebauungsplan Nr. 345. https://www.erlangen.de/desktopdefault. aspx/tabid-1619/3544_read-33125 (letzter Zugriff am 23.03.2020)

Stadt Erlangen (2019): Kleinräumige Sozialstruktur der Stadt Erlangen 2018. https:// www.erlangen.de/Portaldata/1/Resources/o80_stadtverwaltung/dokumente/ statistik/13-4_B_2019_1.pdf (letzter Zugriff am 06.08.2019). 
Umweltbundesamt (2015): Stressreaktion und Herz-Kreislauf-Erkrankungen. https:// www.umweltbundesamt.de/themen/verkehr-laerm/laermwirkung/stressreaktionenherz-kreislauf-erkrankungen\#textpart-1 (letzter Zugriff am 31.03.2019).

Umweltbundesamt (2017): Straßenverkehrslärm. https://www.umweltbundesamt.de/ themen/verkehr-laerm/verkehrslaerm/strassenverkehrslaerm\#textpart-1 (letzter Zugriff am 31.03.2019).

Umweltbundesamt (2018a): Feinstaub-Belastung. https://www.umweltbundesamt.de/ daten/luft/feinstaub-belastung (letzter Zugriff am 31.03.2019).

Umweltbundesamt (2018b): Stickstoffdioxid-Belastung. https://www.umweltbundes amt. de/daten/luft/stickstoffdioxid-belastung (letzter Zugriff am 31.03.2019).

Zehner, Klaus (2004): Sozialräumliche Segregation in London. Ein methodischer Ansatz zur Messung sozialer Disparitäten in einer Global City. In: Raumforschung und Raumordnung $62,4-5,301-308$.

\section{Poor Doors in Erlangen. Environment-related microsegregation under conditions of reurbanisation}

As competition for urban resources intensifies as a result of reurbanisation, segregation processes are becoming increasingly small-scale. A case study in Erlangen shows how planning in the district of Röthelheimpark and Rathenausiedlung creates preferred residential locations, not least due to the establishment of disadvantaged sites. There are phenomena of micro segregation at neighbourhood level which can hardly be empirically recorded using conventional methods of statistical measurement. The topic is particularly explosive due to the fact that health risks are unequally distributed, because environmental pollution is specifically imposed on poorer groups of residents, while environmental resources benefit the wealthy. 